\title{
Multidimensional Risk Management for Underground Electricity Networks
}

\author{
Thalles V. Garcez ${ }^{1}$, Przemysław Szufel ${ }^{2}$ \\ 1 Management Engineering Department, Federal University of Pernambuco, Brazil, \\ tvgarcez@gmail.com \\ 2 Division of Decision Support and Analysis, Warsaw School of Economics, Poland, \\ pszufe@sgh.waw.pl
}

\begin{abstract}
In the paper we consider an electricity provider company that makes decision on allocating resources on electric network maintenance. The investments decrease malfunction rate of network nodes. An accidental event (explosion, fire, etc.) or a malfunctioning on underground system can have various consequences and in different perspectives, such as deaths and injuries of pedestrians, fires in nearby locations, disturbances in the flow of vehicular traffic, loss to the company image, operating and financial losses, etc. For this reason it is necessary to apply an approach of the risk management that considers the multidimensional view of the consequences. Furthermore an analysis of decision making should consider network dependencies between the nodes of the electricity distribution system. In the paper we propose the use of the simulation to assess the network effects (such as the increase of the probability of other accidental event and the occurrence of blackouts of the dependent nodes) in the multidimensional risk assessment in electricity grid. The analyzed effects include node overloading due to malfunction of adjacent nodes and blackouts that take place where there is temporarily no path in the grid between the power plant and a node. The simulation results show that network effects have crucial role for decisions in the network maintenance - outcomes of decisions to repair a particular node in the network can have significant influence on performance of other nodes. However, those dependencies are non-linear. The effects of network connectivity (number of connections between nodes) on its multidimensional performance assessment depend heavily on the overloading effect level. The simulation results do not depend on network type structure (random or small world) - however simulation outcomes for random networks have shown higher variance compared to small-world networks.
\end{abstract}

Keywords: cascade failure, network decision making, underground electricity grids.

\section{Introduction}

The aim of the paper is to propose simulation methods to assess influence of expenditures of network structures on network malfunctions and 


\section{Thalles V. Garcez, Przemystaw Szufel}

multidimensional consequences of those malfunctions. In the paper we consider a decision making problem for expenditures on an electricity network.

Garcez and Almeida (2013) developed a multidimensional assessment tool for measuring the risk in an underground electricity distribution system. The tool ranks the alternatives in a risk hierarchy. In the paper, we expanded that approach with the analysis of network dependencies in a power grid. The need for such analysis arises from the fact that there is a dependency relationship between the vaults (nodes) of an underground electricity distribution system. The large-scale interconnected power grid system has grown into one of the most complex man-made technological networks (Han \& Cao, 2004). With the increasing number of nodes the interaction of the components becomes more complex (Wildberger, 1997). According to Dueñas-Osorio and Vemuru (2009), the infrastructure systems and their flow demands are growing at a rate that is outpacing the efforts to upgrade flow capacity and maintain safety margins. In addition, infrastructure systems are becoming more interdependent and failures within a given system are more likely to impact the performance of other systems.

The use of underground electricity distribution networks is increasing worldwide due to several reasons including increased security, protection from the forces of nature and aesthetic benefits to a city (e.g. see Koch and Carpentier, 1992; Walsh and Black, 2005). Aging of an underground grid, lack of appropriate maintenance and interference from other underground systems have generated many accidental events, thus putting human life in danger, and generating consequences for society, the local distribution company and third parties (e.g. see Koch and Carpentier, 1992; Walsh and Black, 2005). For example, in New York, hundreds of accident events in vaults occur every year, such as fires, explosions, smoke, etc. (Rudin et al., 2012). In the city of Rio de Janeiro (Brazil) in the years 2010 and 2011, there were about 70 events in underground vaults.

These accident events can have various consequences and in different perspectives, such as pedestrian deaths and injuries as a result of the fall of a manhole cover or exposure to incident energy; they scare the local population; fires in nearby locations; disturbances in the flow of vehicular traffic; loss to the company image, operating and financial losses; drop in the availability and reliability rates of the system, etc. (Garcez \& Almeida, 2013).

According to Almeida (2001), the competitiveness of an industrial system is directly related to decision making in maintenance area. In some situations, in the business decision analysis it is necessary to allocate additional or supplementary investments (resources) that the company needs to make in turn to make its system safer and more reliabile. This is hard and 
complex task to decision maker, because the systems grid has large number of vaults that should be considered in the analysis, the existence of the great diversity of hazards that can cause accidents and different consequences. Furthermore, these resources (money, time, work teams, technology, etc.) that the electricity distribution company can make available are limited and scarce, so it is not possible to apply the same amount of resources in all underground vaults (alternatives) - see Garcez and Almeida (2013).

The research methodology in our paper is simulation of a system model - e.g. see Gilbert and Troitzsch (2005) and Law (2007). The system here is understood in classical system approach as a set of interconnected elements which was proposed by Ackoff (1971). Hence, in the paper the system is an electricity grid network with interconnected nodes. Similar approaches can be found in the literature - e.g. Sun (2005) has simulated a small-world model of a power grid and measures the network efficiency on both local and global levels. Dueñas-Osorio and Vemuru (2009) studied the effect of cascading failures in the risk and reliability assessment of complex infrastructure systems. We expand the existing approaches by adding a multidimensional risk measurement and by evaluating the impact of electricity grid structure on its efficiency.

The simulation approach allows us to present consequences arising from accidental events in underground system of energy distribution. For this, the simulation shows to be a useful tool to present to decision maker before they make the in-depth analysis to ranking of the risk, and defined priorities of the alternatives.

Law (2007) notes that a simulation process includes modeling the system, implementation, verification, simulation experiments and metaanalysis of simulation results. In the paper we follow the proposed process. Hence the layout of the paper is as follows. After the introduction, in section 2 model assumptions are discussed. Next, in section 3 an implementation is presented that is followed by description of simulation experiments and results of those experiments. The fourth section is the conclusion.

\section{Network effects for underground electricity distribution}

The goal of this section is to present a conceptual model for electricity distribution grid. The conceptual model presentation is divided into two parts. Firstly, in the subsection 2.1 multidimensional performance measures for electricity grid are proposed. Secondly, in the section 2.2 network dependencies in the model are discussed. 


\subsection{Measuring electricity grid operation}

Considering an electricity grid, specifically an underground system, for each node an accidental event is adversely affected (hazard scenario $\theta$, for example, can be represented by fires, explosions, etc.), there will be consequences for the financial loss.

Let us assume that the total accumulated financial dimension on the time $(t)$ can be estimated by sum of the revenue $\left(R_{\text {total }}(t)=\sum_{i=1}^{q} R_{i}(t)\right)$ from each underground vault $(q)$. When a malfunction occurs a fine of rate $f_{\%}$ is imposed on the grid operator. The actual fine $f_{q}$ is calculated by multiplying the rate by the node revenue that would be achieved in normal operation i.e. $f_{q}=-\left(f_{\%} \cdot R_{q}\right)$. Depending on the physical configuration of the distribution network, and on whether there are alternative ways to isolate the system affected (the one that has crashed), it is likely that the financial consequences will be lower due to the lower impact on revenue, fines and the performance of the system - see Garcez and Almeida (2013).

Garcez and Almeida (2013) note that non-financial consequences of a power grid malfunction play important role and should be considered independently of financial ones. Two important non-financial consequences include a number of people injured $\left(N_{t}\right)$ and operational loss. Let $\left(t_{d_{q}}\right)$ represent downtime of a particular node in an electricity grid. This is measured from the moment that the electric power distribution becomes unavailable to the consumer, until it is restored to the standards required by the regulatory agencies. The probability density function lognormal can be used to calculate the result of the function, which represents the maintainability of the system. However, we can convert this operational loss dimension as a financial loss because during this time of system inactivity, the company ceases to accumulate revenue i.e. $D(t)=-t_{d_{q}} \cdot R(t)$

\subsection{Network effects in electricity grid operation}

$\mathrm{Li}$ and $\mathrm{Lu}$ (2005) propose a method to perform risk assessment of a combinative system of transmission network and substation configurations, and, so, capturing the mutual impacts between the transmission network and substation arrangements.

Before decision making it is necessary to understand the relationship between the various underground vaults, i.e., in view of the network of the system. Several consequences can be influenced by the time and relations between different consequences of the dimensions considered. For example, if we look only at one alternative (one specific underground vault) the revenue will accumulate with the forward of the time (hourly, daily, monthly, etc.), and in the occurrence of an accidental event, this increase in revenue will be 
broken because there will be losses, with respect to different loss dimensions, such as, human loss, financial loss or operation loss, previously detailed.

The Figure 1, presents the value of accumulated revenue $R_{q}$ in relation to occurrence of the accidental event (explosion of the underground vault, for example), which occurs in $t_{e}$ time. After elapsed $t_{D_{q}}$ time (called downtime), in $t_{r}$ time, the system is restored and comes back to work again, accumulating revenue.

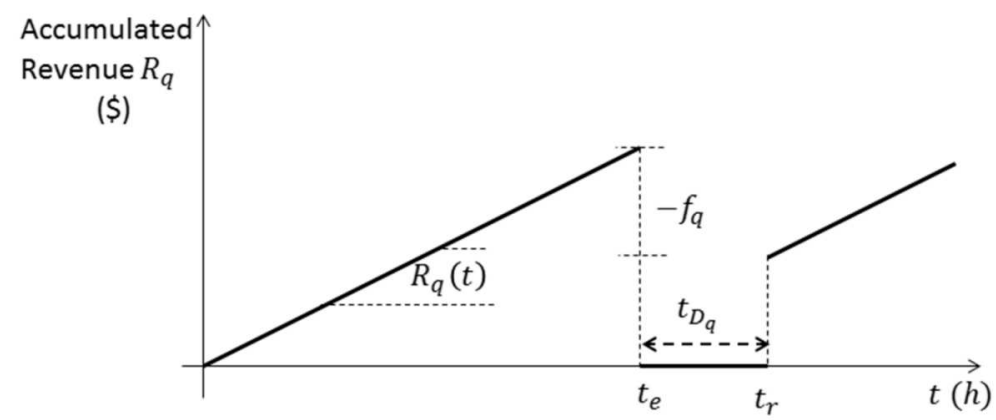

Figure 1. Behavior of the accumulated revenue $R_{q}$ in relation to occurrence of the accidental event

We consider each underground vault $(q)$ to represent a network node. If electricity grid is operating perfectly, there is an accumulation of revenues for each node. However, if any accidental event occurs, there will be losses in revenue resulting from the imposition of fines, and the time at which the system remains inactive (downtime). Also, other nodes that are dependent (they are subsequent to the node where the accidental event occurred) will not accumulate revenue because they are not powered. Moreover the market regulator can impose a fine $b_{q}$ on an electricity grid operator. The fine level $b_{q}$ is the percentage of revenue generated by a node $R(t)$ and i.e. $b_{q}=-b_{\%} R(t)$.

Furthermore, if an accidental event occurs non-financial loss can be generated. In this specific case, we consider the non-financial loss such as injuries to people. Other effects may be caused by an accidental event in an underground vault, that we will call "network effects", which is the increased probability of other accidental events, and the occurrence of blackouts. The malfunction of a highly connected node is likely to result in subsequent malfunctions. Hence, highly connected nodes require greater care in maintenance. Thus, a cascading failure effect can be observed.

Figure 2 represents the relationship between normal operation and the occurrence of an accidental event in each node and the results generated by these relations in a network system, as described above. 


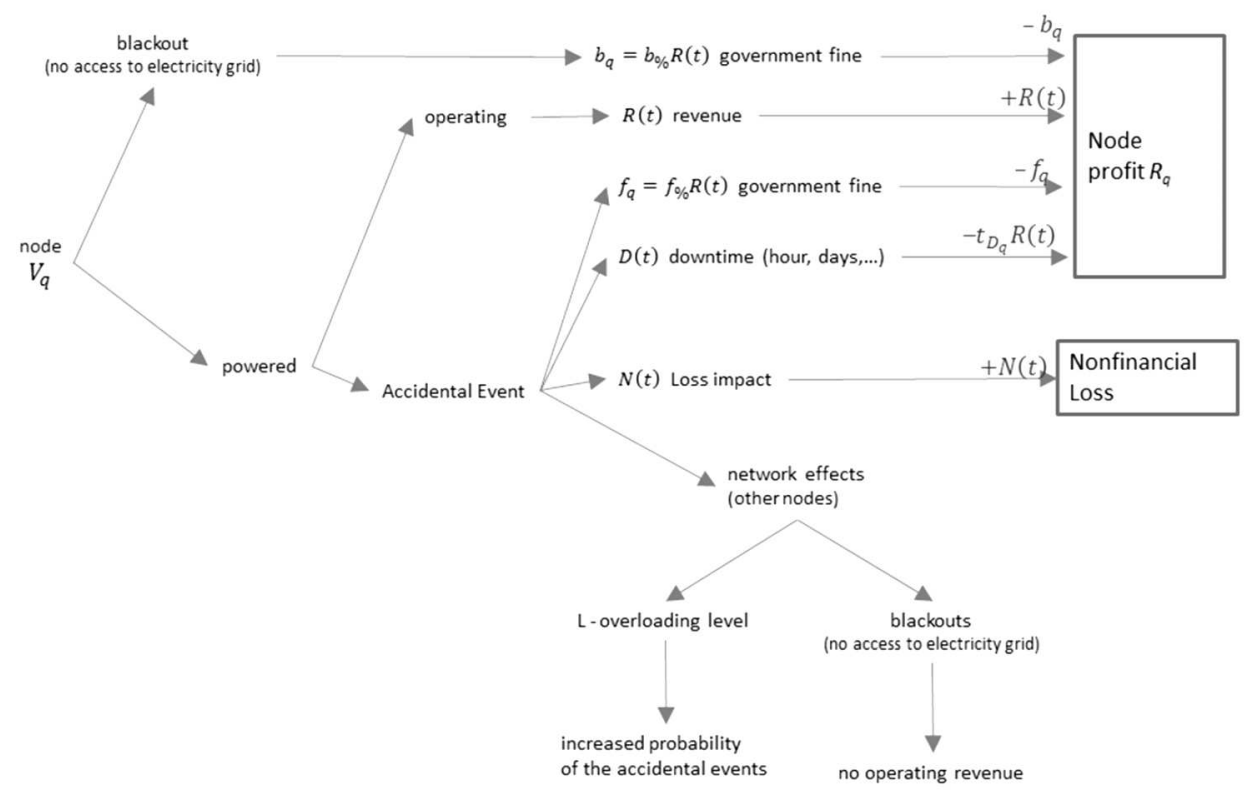

Figure 2. Transitions of node states in an underground electricity network model

The proposed electricity grid management problem has complex, nonlinear dependences between its components. Hence, we apply the simulation approach to analyze it. Applying simulation to complex system analysis is consistent with the literature - e.g. Holland (1992), Law (2007) and Mitchell (2009).

\section{Simulating network effects}

The goal of this chapter is to present the implemented simulation model and results of simulation experiments. The discussion starts with presentation of the simulation model implementation and model configuration. The implementation description is illustrated with screenshots from example simulation runs. Next, experiment design is discussed. Finally the simulation results are presented.

\subsection{Simulation model implementation}

The model has been implemented with the Python programming language. For network manipulation and visualization the networkX library (see Hagberg et al., 2008) has been used. For graphical control of simulation the and WPyCX simulation library has been used. The WPyCX is fork 


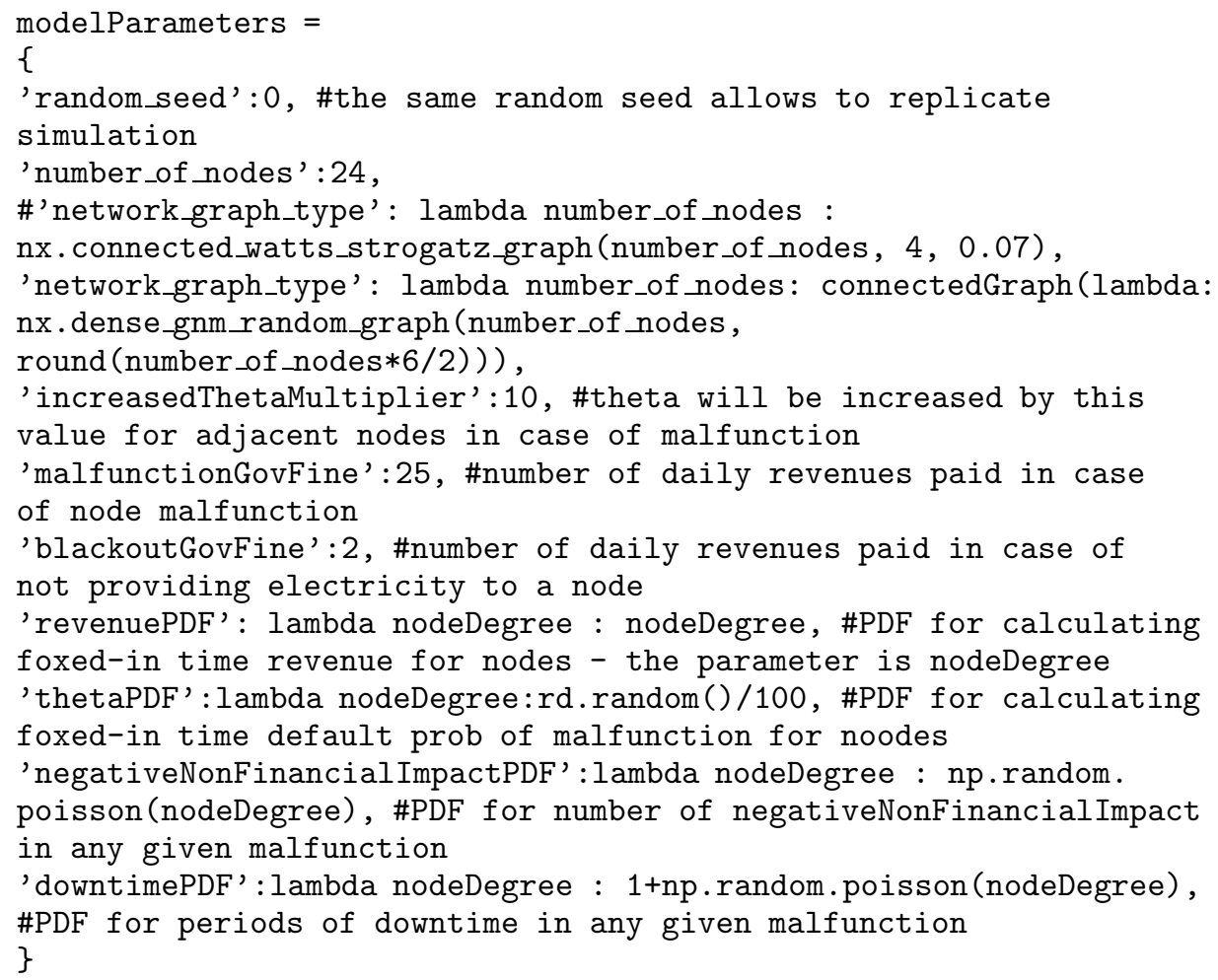

Figure 3. Model parameterization includes the base random seed, number of nodes, network structure, malfunction and downtime probability density function

of the PyCX simulation library. The PyCX was designed by Sayama (2013) while the WPyCX fork has been co-developed by one of the authors.

The main simulation model consists of the three routines: init ()$, \operatorname{step}()$ and draw (). The init() routine is called once per simulation to assign the initial values and generate initial network structure. The step () routing is executed once per each simulation tick in the model. We assume that one time unit (one simulation tick) in the model represents one day in the realworld system. The draw () routine is executed only when simulation is run in graphical mode.

The model parameterization has been presented on Figure 3 and includes the base random seed, number of nodes, network structure, malfunction and downtime probability density function. The random seed value allows to replicate simulation results. The simulated network size (24) includes the power plant factory. The network graph type is configurable as a function - here an anonymous lambda function is being used. In the simu- 


\section{Thalles V. Garcez, Przemystaw Szufel}

lation setup process we only consider connected networks - a path between the factory and each node must exist (however, during a simulation process this part can be temporarily broken due to node malfunction). The fines are defined as multiplier of daily revenues. For the negative nonfinancial impact $N(t)$ and out-of-service days in case of malfunction $d(t)$ probability density functions can be defined.

In the model we use Poisson distribution with parameter dependent on node degree in the network. For each node a malfunction probability is generated. It is assumed that this value is $\theta_{q} \sim U(0,0.01)$. Hence $\theta_{q}$ is uniformly distributed random number from the range $(0,0.01)$.

The simulation model source code can be found in the Appendix. In the next subsection the experiment scenario and parameter sweep will be discussed for the presented set of model parameters.

\subsection{Simulation experiments}

The simulation experiments have been carried out for 24-node networks. In the experiments two different network structure types are considered: small world (see Watts and Strogatz, 2008) and random network (see Erdös and Rényi, 1960). For each network type 3 connectivity levels will be analyzed: 24, 48 and 72 edges - see Table 1 .

The key parameter of the model is the impact of overloading on the performance of adjacent nodes. The overloading influence describes how many times the malfunction probability increases due to overloading. This parameter is further multiplied by the level of node overloading. The experiment factorial design includes 4 different levels of overloading influence levels $1,4,7$ and 10 .

Hence we have 24 possible simulation model configurations. For each of those 24 parameter settings network structure is randomly generated and electricity grid simulation is being run. For each parameterization simulations are replicated 50 times - with 50 different randomly generated networks (however, these networks follow the requested type from table 1). Finally $3 \cdot 2 \cdot 4 \cdot 50=1200$ simulations of electricity grid performance dynamics have been run with each lasting for 1000 periods (days).

Law (2007) points out that using the common random numbers (CRN) technique can improve comparability of simulation outcomes for various scenarios. In the experiments the $\mathrm{CRN}$ was introduced through a common random seed for each of 24 scenarios for a particular run out of 50 repetitions. Hence, the same starting operational parameters (e.g. the base malfunction probability) of nodes are being compared but in different network layouts. 
Table 1

Two network types with 3 different connectivity levels will be analyzed in simulation experiments

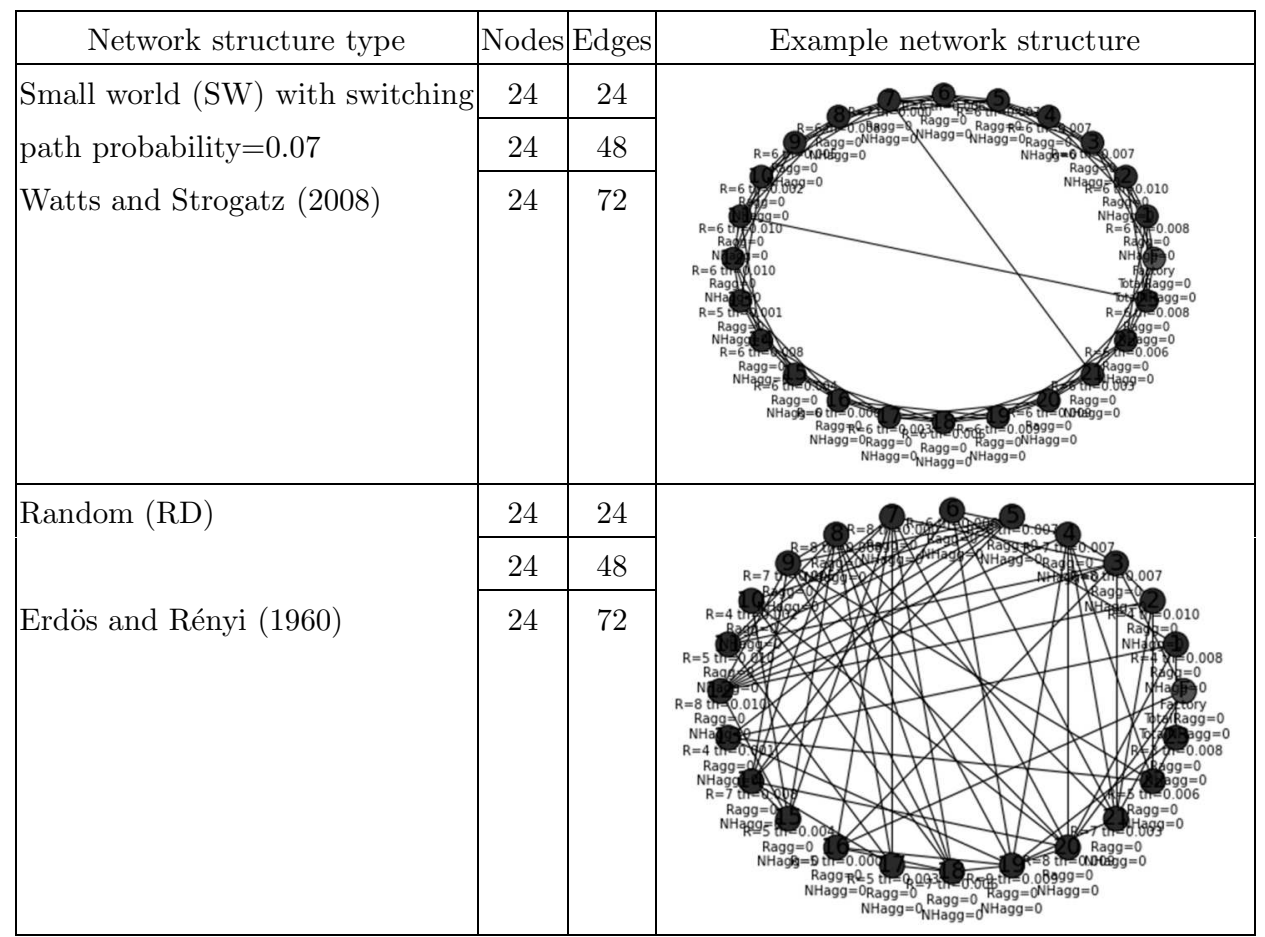

\subsection{Dynamics of electricity grid performance}

The goal of this subsection is to present simulation results. The presentation of results is divided into two parts. Firstly, results of the simulation experiments will be presented. Next, some aspects of the achieved results will be discussed in more detail and will be illustrated with conclusions drawn from single simulation.

The simulation experiments were designed along instructions presented in the subsection 3.2. According to assumption made in the section 2.2, two performance measures of the electricity network are being analyzed: revenue and negative impact (nonfinancial loss). Figure 4 represents an example performance trajectories for all nodes in a network. The simulation has lasted for 1000 periods (see section 3.2) - it can be seen that the node performance varies strongly in the network. Values of node performance factors are being calculated for single nodes in the network and next they are being aggregated for the entire network. The nodes operate for several 


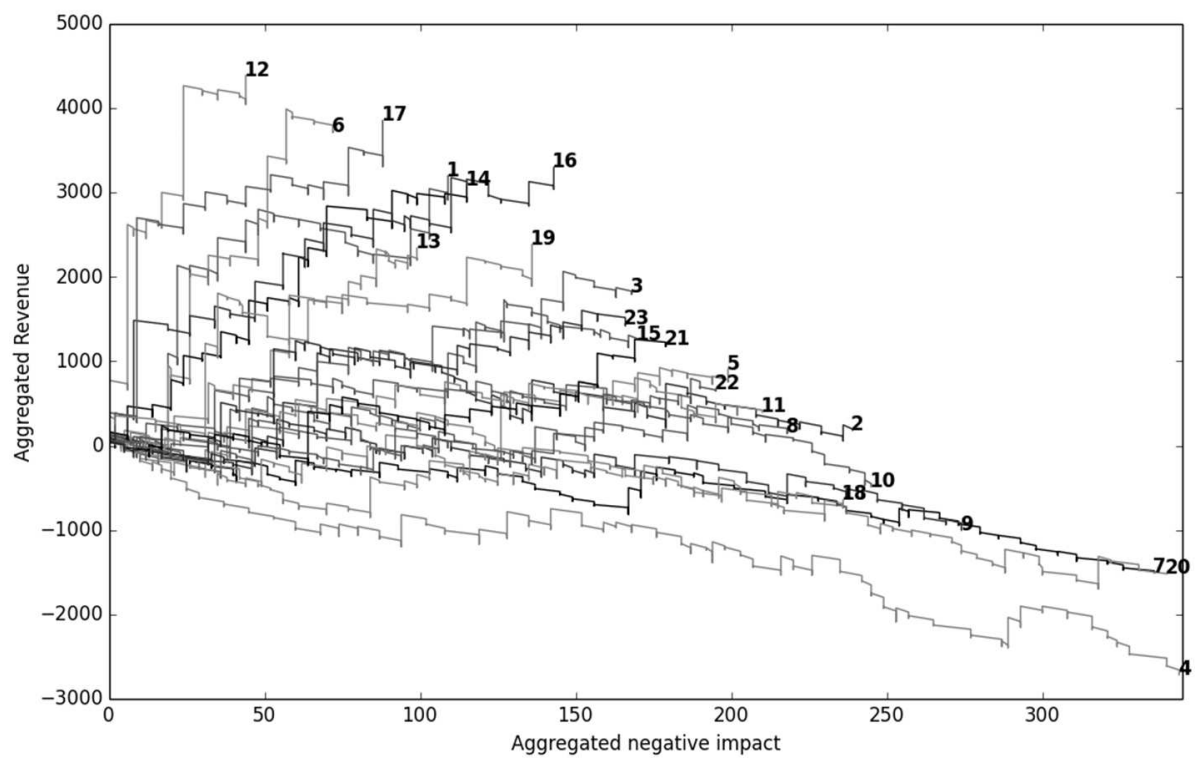

Figure 4. Dynamics of node performance - two outcomes are measured: aggregated revenue and aggregated nonfinancial loss

periods. Hence, the aggregated negative impact can change only with time, while aggregated revenue of a node can change depending on the number of malfunctions the node undergoes.

The simulation experiments results presented on Figure 5 show the important role of impact of network nodes overloading vulnerability and network connectivity (number of edges) on network performance. Each box on the graph has been plotted by calculating results of 50 simulation runs for a given randomized network structure. It can be seen that for networks with small overloading effects connectivity leads to increase in profit and this relationship is independent of the network structure type. In networks with strong overloading the increase in connectivity first leads to increase of performance while later it leads to decrease of profit.

Figure 6 presents dependencies between network connectivity and negative impact (nonfinancial loss). It can be seen that with higher connectivity the negative impact increases. However the increase is much faster in networks with higher overloading effect. Hence, the simulation results show that overloading effect is important factor for network performance and dependence between the performance and the structure.

The overloading effect has very strong effect on performance because of possibility of cascade failure. A node malfunction increases the probability of malfunction for other nodes due to overloading (see the section 2.2). 

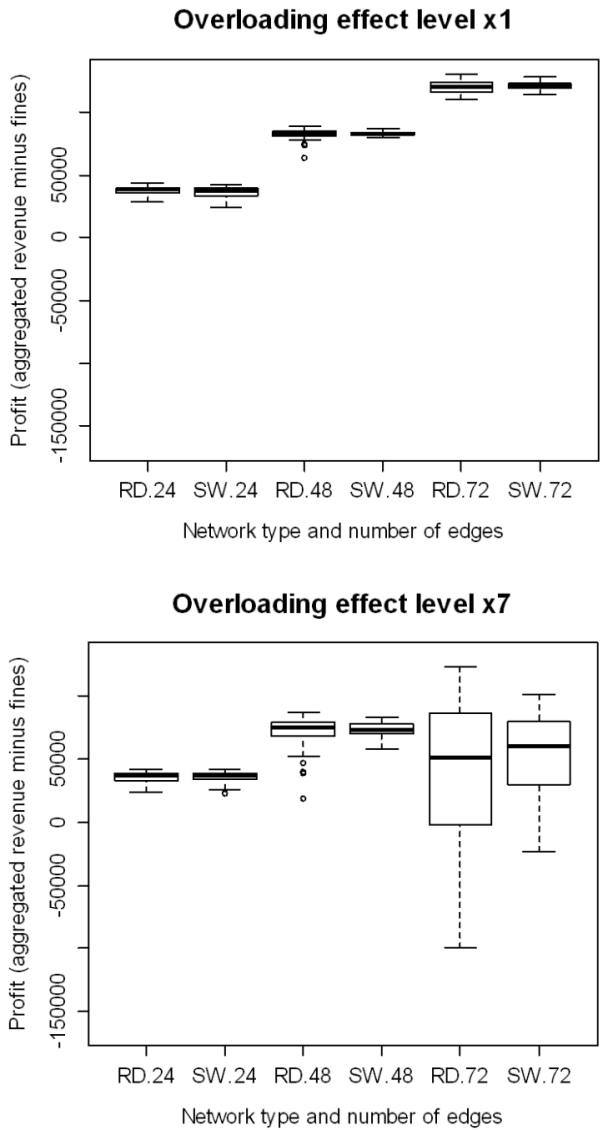
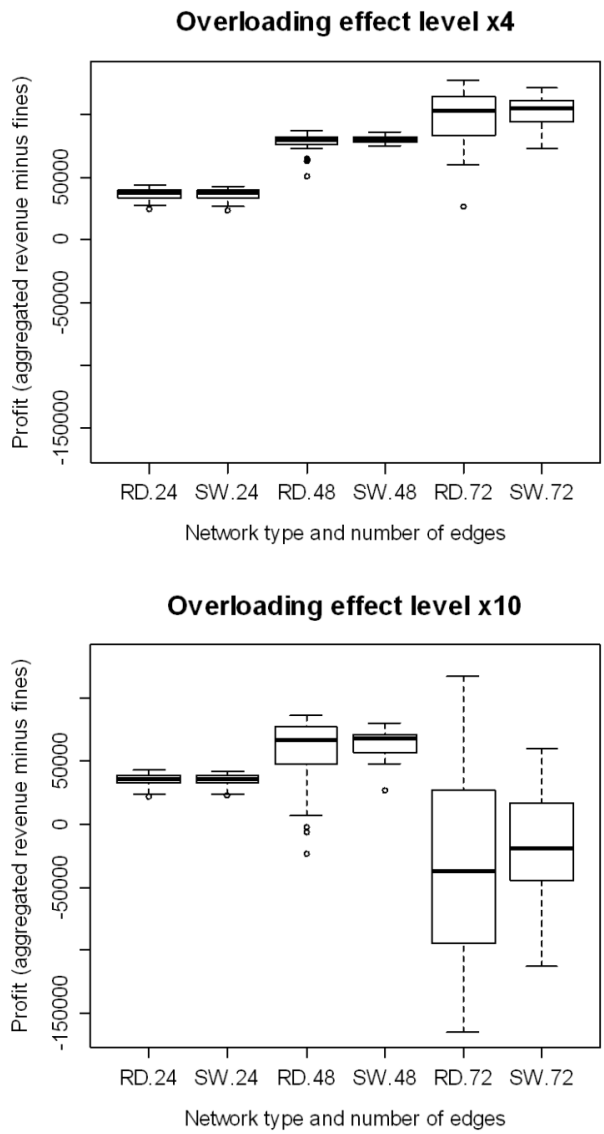

Figure 5. Changes in effect level for node overloading influence the shape of dependence between connectivity of the network and aggregated profit. This dependence is independent of network type $[\mathrm{RD}=$ Random, SW $=$ Small world $]$

Overloading effect level x1

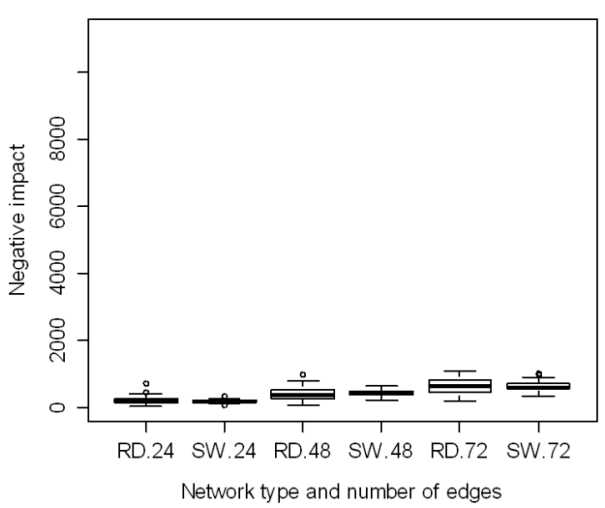

Overloading effect level x4

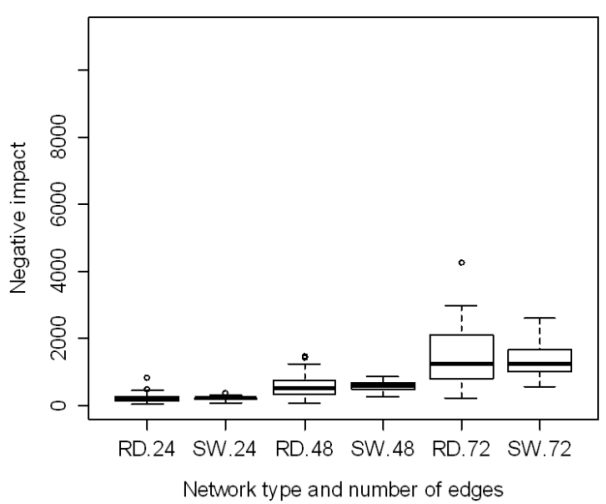



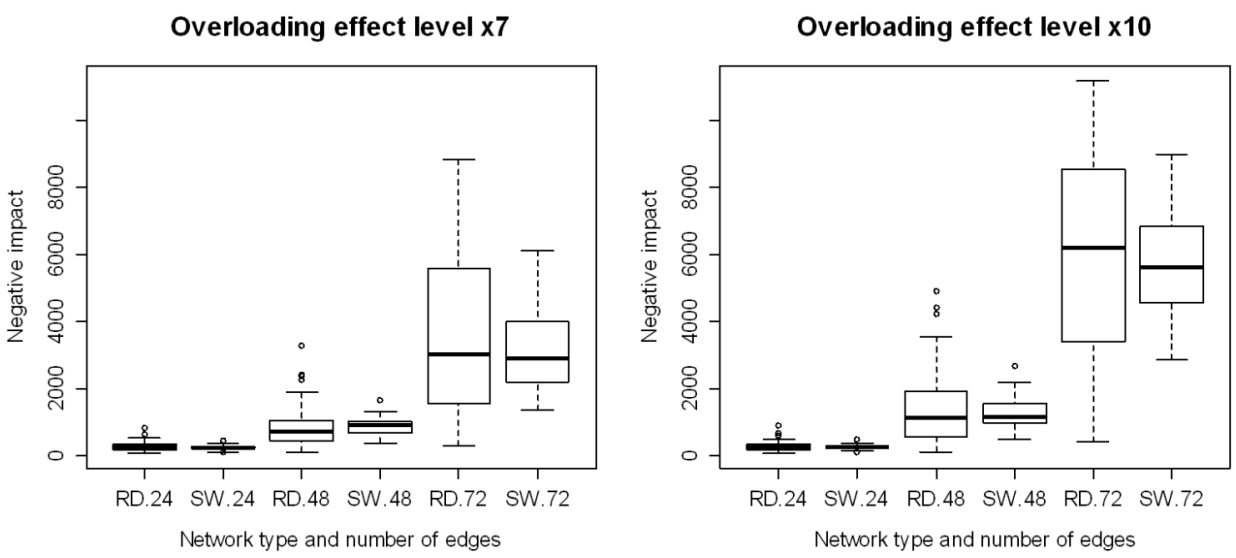

Figure 6. Network connectivity increases the average negative impact of the network (i.e. number of malfunctions) - this increase is higher with higher overloading effect

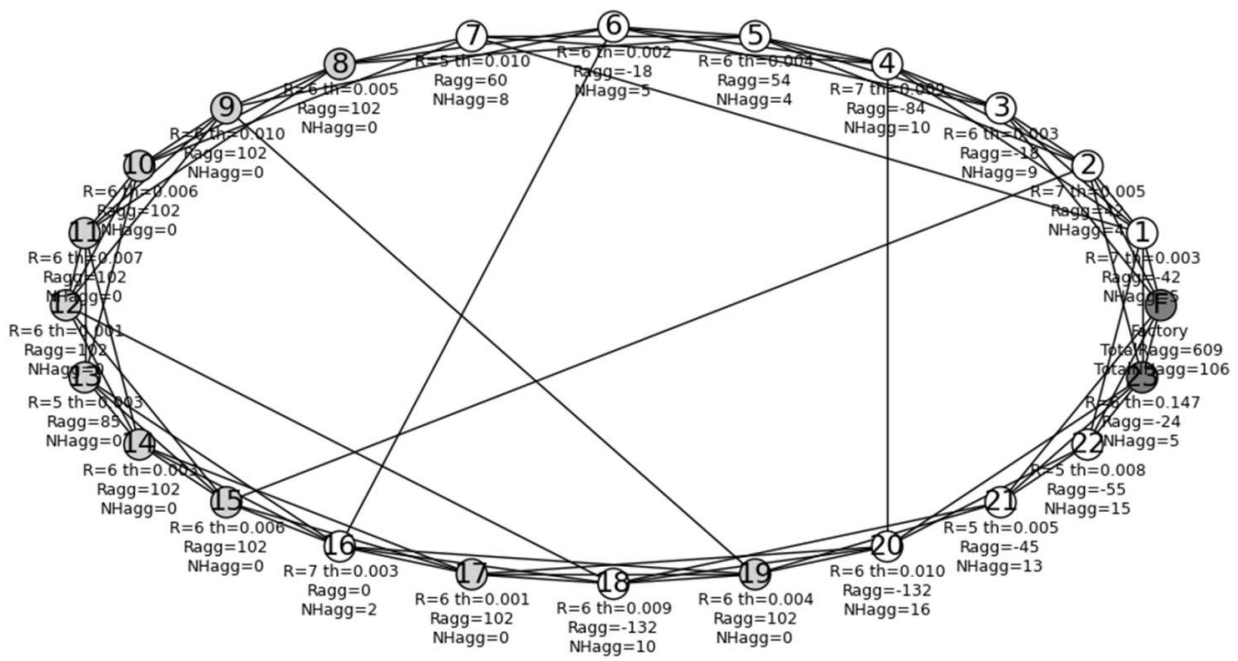

Figure 7. An example cascade failure with a blackout in a small world network. The gray color represents the electricity power plant $(F)$ and a powered node, the white color represents node malfunction while the light gray represents power shortage (blackout)

In high-connectivity networks this situation can lead to a cascade failure in electricity grid. A sample cascade failure for a network has been presented in figure 7.

The electricity grid operator can make decisions to repair a particular node. The repair leads to a decrease of malfunction rate for that node. It has also been remarked that due to the overloading effect, repairing a node 
has also a significant impact on other adjacent nodes. Let's consider a damaged electricity network bringing loss with every time period presented on Figure 8. The figure presents dynamic path for profit and negative impact for each node for the period of 1000 days. The graph a) presents performance of network nodes with no budget allocated for repairs. The highest performance is of nodes 6,12 and 17 while nodes 4, 7, 9, 10, 18 and 20 create excessive volume of negative impact and probably a decision to repair those nodes should be made. For the network we consider a decision to repair nodes 7,9 and 18. It can be seen that such decision leads to structural changes in performance of several other nodes in the network - e.g. the performance of node 4 sharply increases. This effect is caused by the fact that malfunction of a node leads to decreased performance of adjacent nodes.

a) no funds allocated on repair

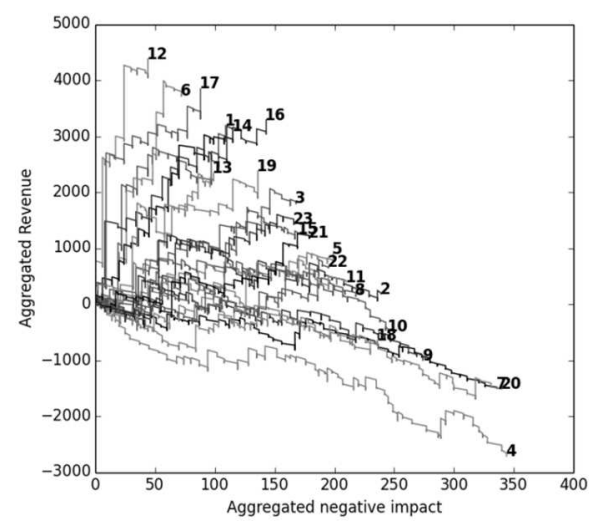

b) nodes 7,9 and 18 repaired

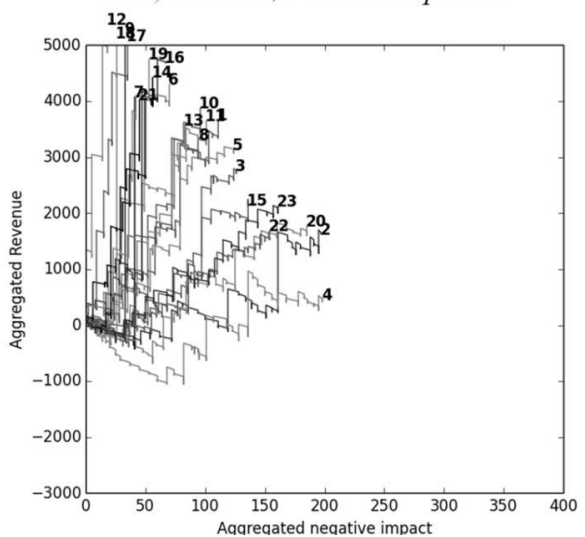

Figure 8. The network effect on of repairing nodes - performance of other nodes (e.g. 4 has increase)

The presented simulation results lead to the following four conclusions. Firstly, the interference level between nodes strongly influences performance of the nodes - especially for networks with high connectivity. This effect can be observed regardless of the network structure type (random or small world). Secondly, the independence between network structure type and network performance means that computer simulation of artificial networks can be applied to real world networks having different structures. Thirdly, maintenance decisions on the network should depend not only on performance of a single node but the effect on entire network performance should be simulated. Finally, the variance of network efficiency is much higher for random networks than for small world networks. 


\section{Conclusions}

The network structures of power distribution systems are critical to society. Furthermore, these structures are large and complex, with large amounts of connections (nodes) and it requires more dedication from the company concerning proper maintenance and risk management, in order to maintain the reliability and safety of the system at levels acceptable to society. Thus, decision making to prioritize resources to apply in the system become a complex and arduous task for the decision maker.

As noted, it is so important to perform multidimensional risk assessment, as evidenced by Garcez \& Almeida (2013), as well as assessing influence of network effects on electricity system. Therefore, the use of simulation is an appropriate tool that allows managers to understand the interactions between various nodes in the system and to verify the system's behavior over time, and, thus, allowing the decision maker to have more information to take the right steps.

The simulation results show that network effects have crucial role for decisions in the network maintenance - outcomes of decisions to repair a particular node in the network can have significant influence on performance of other nodes. However, those dependencies are non-linear. The effects of network connectivity (number of connections between nodes) on its multidimensional performance assessment depend heavily on the overloading effect level. The simulation results do not depend on network type structure (random or small world) - however simulation outcomes for random networks have shown higher variance compared to small-world networks.

The proposed approach was illustrated with an electricity grid network. However it can also be applied to analysis of different distribution systems (natural gas, oil and water). Moreover it can be applied to dynamic analysis of other complex systems where a malfunction of a single node can increase probability of malfunction of adjacent nodes. This includes financial systems (including bank networks) and transportation systems (airborne and ground).

The further research in the presented area includes applying multicriteria decision making methods that incorporates structure preference of the decision maker and it treats the uncertainties inherent of the context. Another area of research is expanding the simulation scenarios into different network structures and network parameterizations. 


\section{Appendix - the power grid simulation model}

The model has been programmed with the Python programming language with the PyCX simulation library proposed by Sayama (2013). Below only the computational part of the model is presented - the visualization and the parameter sweep parts of the source have been excluded due to space limits.

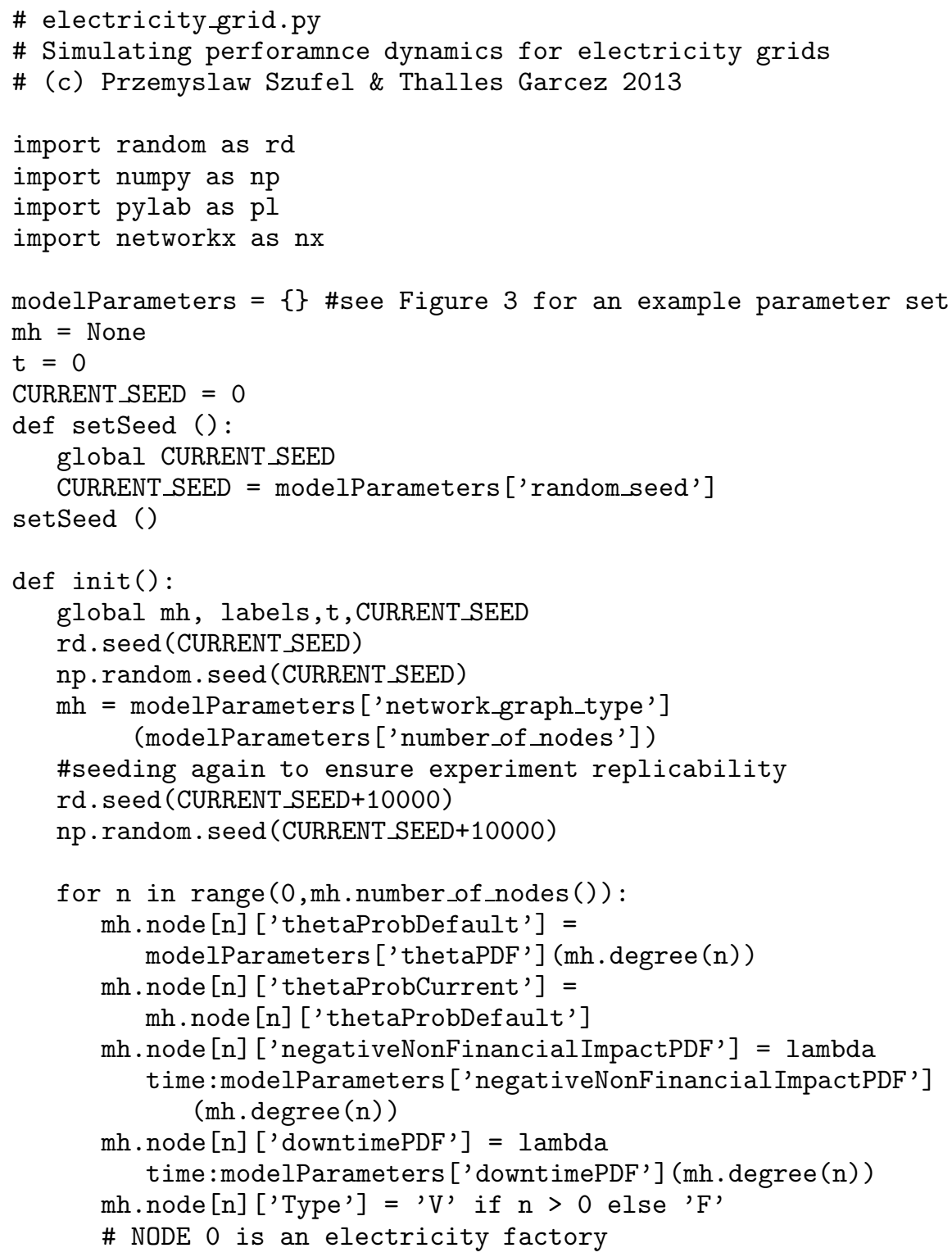


$\operatorname{mh}$. node [n] ['Revenue'] = modelParameters ['revenuePDF'] (mh.degree (n))

$\mathrm{mh}$. node [n] ['Broken'] =0

mh.node [n] ['OverloadLevel'] = 0

\# a node is overloaded when adjacent nodes brake

mh.node [n] ['WillBeRepairedAtPeriod'] = None

\#Aggregetes

mh.node [n] ['RevenueAggregated'] $=0$

mh.node [n] ['NegativeNonFinancial ImpactAggregated'] $=0$

\#histories of aggregates

$\mathrm{mh}$. node [n] ['RevenueAggHistory'] = []

mh.node [n] ['NegativeNonFinancialImpactAggHistory'] = []

try:

$\operatorname{mh}$. node [n] ['Powered'] $=1$

if $\mathrm{nx}$.shortest_path_length $(\mathrm{mh}, 0, \mathrm{n})>0$ else 0

except $\mathrm{nx}$. NetworkXNoPath:

$\mathrm{mh}$. node $[\mathrm{n}]$ ['Powered'] $=0$

$t=0$

CURRENT_SEED += 1 \#Increase the seed for subsequent runs

def $\operatorname{draw}()$ :

pass

\# The visualistion code has been excluded due to space limits def $\operatorname{step}()$ :

global $t$

graphChange $=$ False

if 'repairNodesTime' in modelParameters

and modelParameters ['repairNodesTime'] [1]==t:

for $\mathrm{n}$ in modelParameters ['repairNodesTime'] [0]:

mh. node [n] ['Broken'] =0

$\operatorname{mh}$. node $[\mathrm{n}]$ ['thetaProbDefault'] $=0$

mh. node $[\mathrm{n}]$ ['thetaProbCurrent'] $=0$

\#track status change of nodes

for $\mathrm{n}$ in range( $1, \mathrm{mh}$.number_of nodes ()):

if (mh.node $[\mathrm{n}][$ 'Broken']==0):

\#Only a powered node can break

if (mh.node [n] ['Powered'] ==1

and $r d . r a n d o m()<\operatorname{mh}$. node [n] ['thetaProbCurrent']):

\#transformator has exploded

mh. node [n] ['Broken'] =1

mh.node [n] ['WillBeRepairedAtPeriod'] = $t+m h . n o d e[n]$ ['downtimePDF'] $(t)$;

\#need to pay fine

$\mathrm{mh}$. node [n] ['RevenueAggregated'] -= mh.node [n] ['Revenue']* modelParameters ['malfunctionGovFine']

\#some people are negativeNonFinancialimpact

mh.node [n] ['NegativeNonFinancial ImpactAggregated']+= $\mathrm{mh}$.node [n] ['negativeNonFinancialImpactPDF'] (t)

graphChange $=$ True 


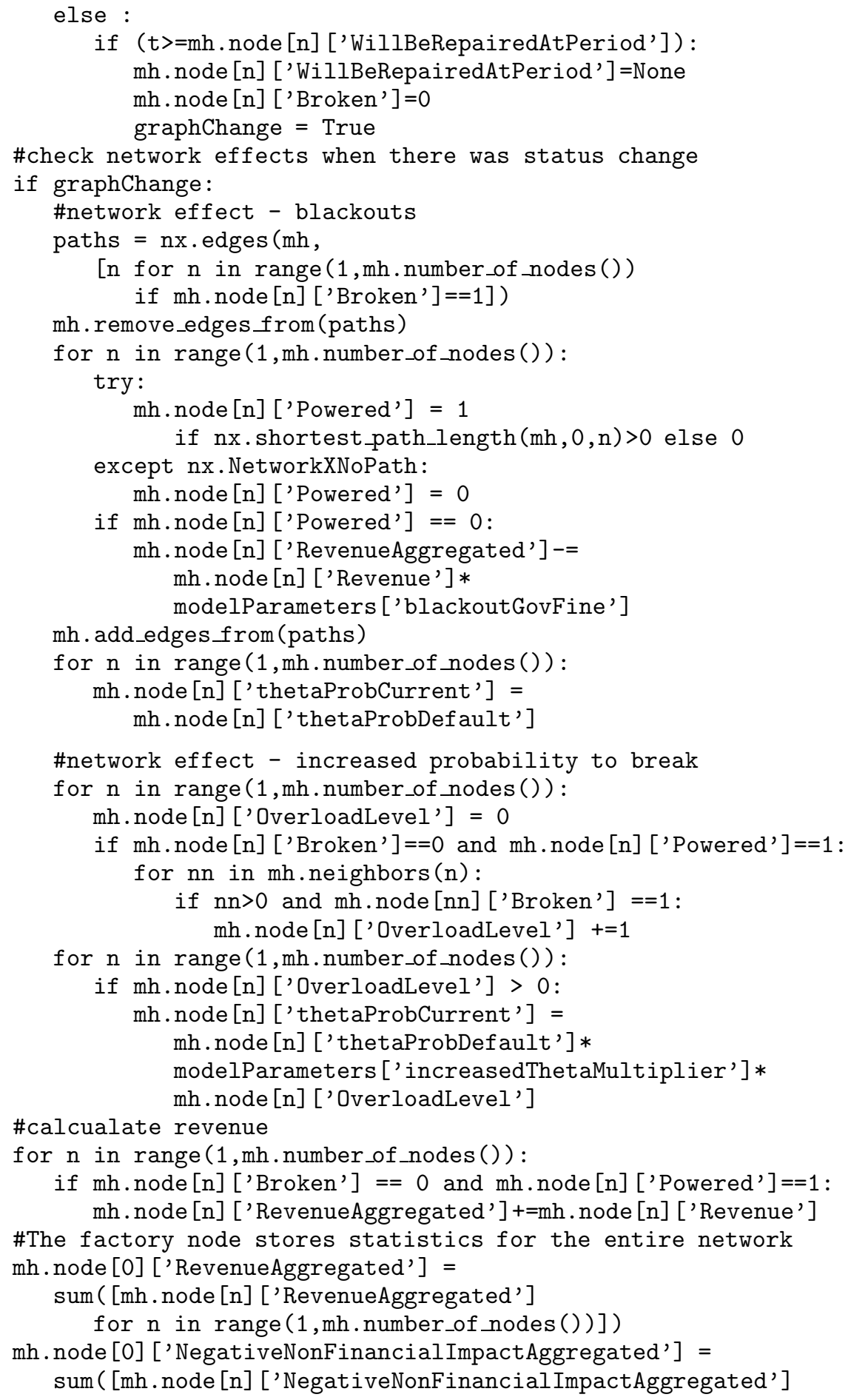




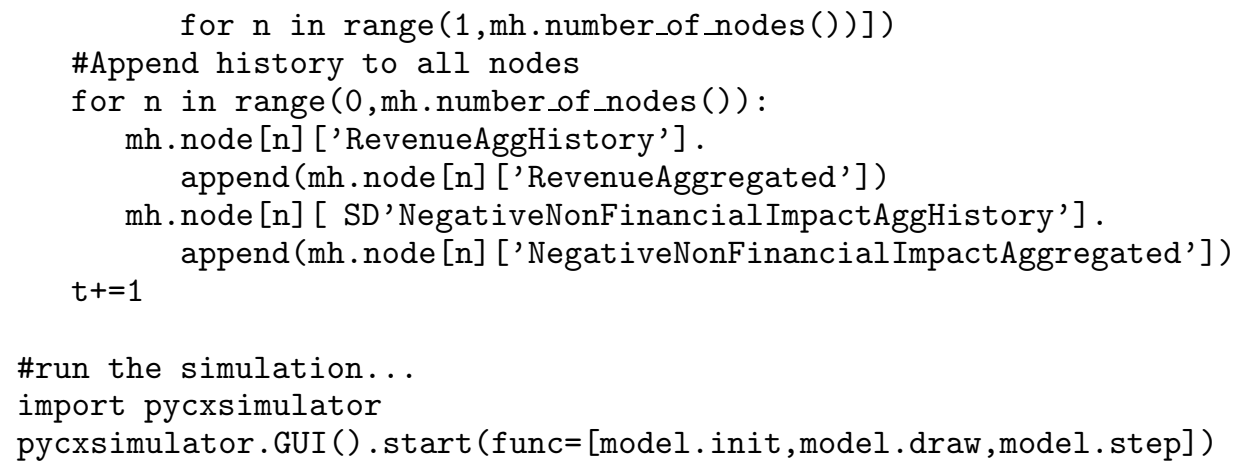

\section{R E F E R E N C E S}

Ackoff, R. L. (1971). Towards a system of systems concepts. Management science $17(11), 661-671$.

Almeida, A. T. de (2001). Multicriteria decision making on maintenance: Spares and contracts planning. European Journal of Operational Research, 129, 235241.

Dueñas-Osorio, L. and Vemuru, S. M. (2009). Cascading failures in complex infrastructure systems. Structural Safety 31, 157-167.

Erdös P. \& Rényi A. (1960). On the evolution of random graphs. Publications of the Mathematical Institute of the Hungarian Academy of Sciences, 5, 17-61.

Garcez, T. V., \& Almeida, A. T. de. (2013). Multidimensional Risk Assessment of Manhole Events as a Decision Tool for Ranking the Vaults of an Underground Electricity Distribution System. IEEE Transactions on Power Delivery, 1-1. doi:10.1109/TPWRD.2013.2273083

Gilbert, N., \& Troitzsch, K. G. (2005). Simulation for the social scientist, McGrawHill International.

Hagberg A. A., Schult D. A, Swart P.J. (2008). Exploring network structure, dynamics, and function using NetworkX. Proceedings of the 7th Python in Science Conference (SciPy2008), Pasadena, CA USA, 11-15.

Han, Z. X., \& Cao, Y. J. (2004). Power system security and its prevention. Power System Technology, 28, 1-6.

Holland, J.H. (1992). Complex Adaptive Systems, Daedalus, 121(1), 17-30.

Keeney, R.L., Raiffa, H. (1976). Decision With Multiple Objectives: Preferences and Value Trade-offs. New York: Wiley.

Koch, B., \& Carpentier, Y. (1992). Manhole explosions due to arcing faults on underground secondary distribution cables in ducts. IEEE Transactions on Power Delivery, 7(3), 1425-1433. doi:10.1109/61.141861

Law, A. M. (2007). Simulation modeling and analysis, Boston, MA: McGraw-Hill Education. 
Li, W., \& Lu, J. (2005). Risk Evaluation of Combinative Transmission Network and Substation Configurations and its Application in Substation Planning. IEEE Transactions On Power Systems, 20(2), May 2005.

Mitchell M. (2009). Complexity - a guided tour, New York: Oxford University Press.

Python Software Foundation. Python Language Reference, version 3.3. Available at http://www.python.org.

Rudin, C., Waltz, D., Anderson, R. N., Boulanger, A., Salleb-Aouissi, A., Chow, M., ... Wu, L. (2012). Machine learning for the New York City power grid. IEEE transactions on pattern analysis and machine intelligence, 34(2), 328-45. doi:10.1109/TPAMI.2011.108

Sayamam H. (2013). PyCX: a Python-based simulation code repository for complex systems education, Complex Adaptive Systems Modeling 2013, 1:2.

Sun, K. (2005). Complex Networks Theory: A New Method of Research in Power Grid. IEEE Conference \& Exhibition: Asia and Pacific Dalian, China.

Walsh, B. P., \& Black, W. Z. (2005). Thermodynamic and Mechanical Analysis of Short Circuit Events in an Underground Vault. IEEE Transactions on Power Delivery, 20(3), 2235-2240. doi:10.1109/TPWRD.2005.848445

Watts D.J. and Strogatz S.H. (1998). Collective dynamics of 'small-world' networks, Nature, 393, 440-442

Wildberger, A. M. (1997). Complex adaptive systems: concepts and power industry applications. IEEE Control Systems, 17, 77-88 